\title{
A search for new light charged particles in photoproduction
}

\author{
V.I. Alekseev ${ }^{1}$, V.A. Baskov ${ }^{1}$, V.A. Dronov ${ }^{1}$, Yu.F. Krechetov ${ }^{2}$, A.I. L’vov ${ }^{1, *}$, \\ L.N. Pavlyuchenko ${ }^{1}$, V.V. Polyanskiy ${ }^{1}$, and S.S. Sidorin ${ }^{1}$ \\ ${ }^{1}$ P.N. Lebedev Physical Institute, Moscow, Russia \\ ${ }^{2}$ Joint Institute for Nuclear Research, Dubna, Russia
}

\begin{abstract}
Motivated by recent V.A. Nikitin's reports on observation of new light charged particles with a 2-meter JINR propane bubble chamber we perform an experiment at the LPI electron synchrotron "Pakhra" with the aim to detect such particles in the Bethe-Heitler process. Theoretical limits for masses of new light charged particles of spin $0,1 / 2$ and 1 arising from precise data on the muon anomalous magnetic moment are recalculated and updated. A geometry of the photoproduction experiment is proposed that optimizes signal-tonoise ratio. First results of the experiment are exposed.
\end{abstract}

\section{Introduction}

This work was motivated by recent sensational statements made by Prof. V.A. Nikitin (JINR) at several seminars and public lectures [1]. The main statement was that on 7000+ stereo photos made with the JINR 2-meter propane bubble chamber irradiated by $10-\mathrm{GeV}$ protons of the JINR synchrophasotron there were identified a few tracks of unusual charged particles $\ell^{ \pm}$of the mass near $9 \mathrm{MeV}$. Presumably, these particles $\left(\ell^{ \pm}\right)$, which V.A. Nikitin called «anomalous leptons» due to some guesses concerning mass spectrum of leptons in the Standard Model and beyond, are produced in the electromagnetic conversion reactions $\gamma \rightarrow \ell^{+}+\ell^{-}$caused by photons from decays of $\pi^{0}$ generated in strong interactions of the proton beam with the substance of the bubble chamber.

Examples of anomalous tracks observed by V.A. Nikitin are shown in Fig. 1. ${ }^{1}$ The key point in the analysis of the tracks is determination of the particle mass that is performed by measuring the particle momentum $p$ (via measuring a curvature of the trajectory in a known magnetic field) and the particle kinetic energy $T$ (via measuring range of the particle in propane). Because of fluctuations in the energy losses in substance measurement of $T$ is the most delicate part of the whole procedure of reconstruction of the mass. Nevertheless V.A. Nikitin successfully demonstrated that it works quite well in the case of known tracks of electrons and muons.

In total he found 9 anomalous tracks (of positive and negative electric charge) with the average particle mass $9.4 \pm 2 \mathrm{MeV}$ and momenta in the range $35-95 \mathrm{MeV}$. The total length of the 9 selected tracks seen in the optical window of the bubble chamber is $312 \mathrm{~cm}$, and two

\footnotetext{
*e-mail: 1vov@lebedev.ru

${ }^{1}$ Authors thank V.A. Nikitin for a permit to briefly expose here and below some of his findings.
} 


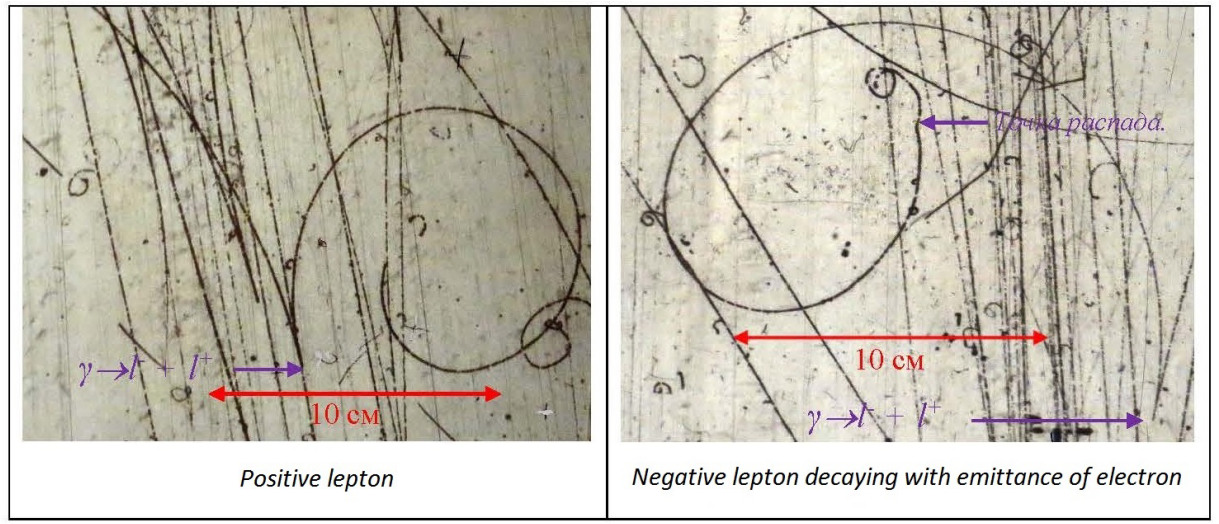

Figure 1. Examples of tracks of «anomalous leptons» found in the propane bubble chamber [1]

spots of particle decay to electron/positron are seen on that length. We interpreter this fact as an indication of a rather short life time of the anomalous particles (of order $1 \mathrm{~ns}$ ).

Due to seen decays like $\ell \rightarrow e+$ neutral, where the unidentified neutral state might be, for example, neutrino or photon, one can anticipate that spin of the new particle is probably equal to $S=0, \frac{1}{2}$, or 1 .

One can ask how it is possible that new charged particles (if exist) remained unobserved till now despite they can be easily produced in electromagnetic processes like ordinary electrons and positrons. One should keep however in mind that, for example, the cross section of $\ell^{+} \ell^{-}$pair production by photons in the Coulomb field of nuclei is kinematically suppressed in comparison with the cross section of $e^{+} e^{-}$production by the bigger mass of the anomalous particles,

$$
\frac{\sigma\left(\ell^{+} \ell^{-}\right)}{\sigma\left(e^{+} e^{-}\right)} \sim\left(\frac{m_{e}}{m_{\ell}}\right)^{2} \sim \frac{1}{340},
$$

so it is not so easy to notice small deviations of the photoabsorption cross section from predictions of pure QED, especially if the new particles are unstable and cannot be accumulated in the substance.

Nevertheless general restrictions to the new particles certainly exist, and now we consider one of them related with precision data on the anomalous magnetic moment of the muon.

\section{New particles and anomalous magnetic moment of the muon}

Nowadays anomalous magnetic moment of the muon is measured very precisely [2] and this opens rich possibilities for testing the Standard Model and finding New Physics.

In QED a point-like (Dirac) charged spin $\frac{1}{2}$ particle, due to interactions with fluctuations of quantum fields, acquires an additional (anomalous) magnetic moment $a=(g-2) / 2$. To leading order in the electromagnetic coupling $\alpha=1 / 137.035999049$ (90) [2] this magnetic moment equals $a^{[\mathrm{LO}]}=(\alpha / 2 \pi)$ and is determined by a single loop diagram shown in Fig. $2 \mathrm{a}$. Due to fantastic theoretical work all higher order QED corrections to $a$ up to order $(\alpha / \pi)^{5}$, electroweak corrections, corrections for strong interactions (hadron polarization of vacuum) and light-by-light scattering corrections are nowadays calculated, so the difference $\Delta a$ be- 
tween experimental and theoretical (in the Standard Model) values of $a$ equals [2]

$$
\begin{aligned}
a^{\exp } & =0.00116592091\left(54_{\text {stat }}\right)\left(33_{\text {sys }}\right), \\
a^{\mathrm{SM}} & =0.00116591823\left(1_{\text {weak }}\right)\left(34_{\text {LO hadr }}\right)\left(26_{\text {NLO hadr }}\right), \\
\Delta a & =a^{\exp }-a^{\mathrm{SM}}=\left(2.68 \pm 0.63_{\exp } \pm 0.43_{\text {th }}\right) \times 10^{-9}
\end{aligned}
$$

It is fantastically small but still leaves some small space for additional contributions to the theoretical value, a contribution of «anomalous leptons» being among them.

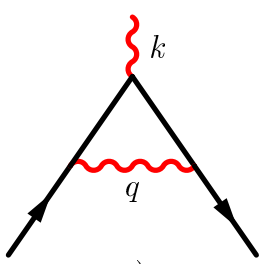

a)

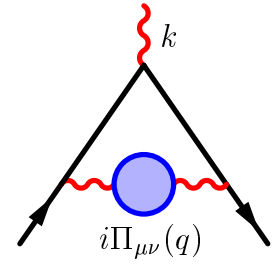

b)

Figure 2. Contributions to the muon anomalous magnetic moment $a$ : a) Leading order, b) hadron vacuum polarization. «Anomalous leptons» can contribute through the diagram $b$ )

«Anomalous leptons» can contribute to $a$ via the diagram in Fig. 2b. The photon polarization operator

$$
\Pi_{\mu v}(q)=-\left(q_{\mu} q_{v}-q^{2} g_{\mu \nu}\right) \Pi\left(q^{2}\right)
$$

that modifies the photon propagator like

$$
\frac{-i}{q^{2}} \rightarrow \frac{-i}{q^{2}}+\frac{-i}{q^{2}} i q^{2} \Pi \frac{-i}{q^{2}}+\ldots=\frac{-i}{q^{2}(1-\Pi)}, \quad \Pi(0)=0
$$

leads to the contribution to the muon anomalous magnetic moment that is schematically given by a loop integral

$$
\text { a.m.m. }=\int \frac{d^{4} q}{(2 \pi)^{4}} \frac{q^{2} \Pi}{q^{2} q^{2}}(\Gamma S \Gamma S \Gamma)
$$

involving three muon vertices $\Gamma$, two muon propagators $S$, one factor of $q^{2} \Pi\left(q^{2}\right)$ and two photon propagators. If $\Pi\left(q^{2}\right) / q^{2}$ vanishes at $q^{2} \rightarrow \infty$ and obeys the unsubtracted dispersion relation

$$
\frac{\Pi\left(q^{2}\right)}{q^{2}}=\frac{1}{\pi} \int \frac{\operatorname{Im} \Pi(t)}{t} \frac{d t}{t-q^{2}-i 0},
$$

the integral for $a$ can be expressed through imaginary part of $-\operatorname{Im} \Pi(t)$, that is positive due to unitarity, in a very simple form:

$$
\text { a.m.m. }=-\frac{1}{\pi} \int \frac{\operatorname{Im} \Pi(t)}{t} K(t) d t
$$

where

$$
K(t)=\frac{\alpha}{\pi} \int_{0}^{1} \frac{x^{2}(1-x) d x}{x^{2}+\left(t / m_{\mu}^{2}\right)(1-x)}, \quad K(t)>0 .
$$

This technique [3] allows to evaluate some contributions of «anomalous leptons» to the muon a.m.m. 
In case of «anomalous leptons» of spin 0 or spin $\frac{1}{2}$ the polarization operator $\Pi(t) / t$ vanishes at infinity and

$$
\begin{array}{ll}
\operatorname{spin} 0: & -\operatorname{Im} \Pi(t)=\frac{\alpha}{12}\left(1-\frac{4 M^{2}}{t}\right)^{3 / 2}, \\
\operatorname{spin} 1 / 2: & -\operatorname{Im} \Pi(t)=\frac{\alpha}{3}\left(1-\frac{4 M^{2}}{t}\right)^{1 / 2}\left(1+\frac{2 M^{2}}{t}\right) .
\end{array}
$$

Then the described formalism is applicable and a straightforward evaluation of the integral for $a$ gives results shown in Fig. 3, in full agreement with [4].

The restriction $a<\Delta a$ in Eq. (1) leads to the $2 \sigma$ bounds on the «lepton» mass,

$$
\begin{aligned}
& \text { «leptons» of spin } 0: \quad M>190 \mathrm{MeV}, \\
& \text { «leptons» of spin } 1 / 2: M>550 \mathrm{MeV},
\end{aligned}
$$

which are not compatible with V.A. Nikitin's observations.

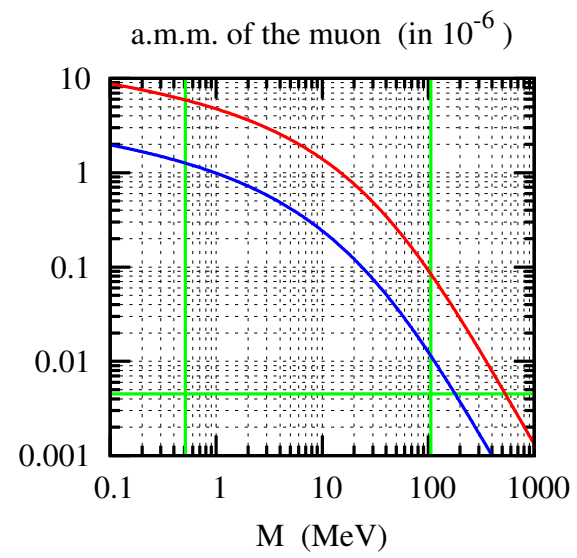

Figure 3. Contributions to the muon anomalous magnetic moment $a$ of «anomalous leptons» of the mass $M$. Blue curves: «leptons» of spin 0 . Red curves: «leptons» of spin 1/2. Vertical line corresponds the muon mass. Horizontal line marks the limit permitted by Eq. (1) for $\Delta a$

A theoretically less clear situation emerges for «leptons» of spin 1 for which we used a Proca vector model. In this case

$$
\text { spin } 1: \quad-\operatorname{Im} \Pi(t)=\frac{\alpha}{12}\left(1-\frac{4 M^{2}}{t}\right)^{3 / 2}\left(3+\frac{t}{M^{2}}\right)
$$

and the integral (2) diverges. Actually, the used Proca effective vector model is unrenormalizable and the loop integral becomes determined by unknown ultraviolet asymptotics of the model.

We can cautiously conclude that cannot fully exclude the possibility to interpreter V.A. Nikitin's «anomalous leptons» as vector particles. A further study of the photon polarization operator is probably needed at $q$ in the region of tens and hundred $\mathrm{MeV}$ in order to draw a more definite conclusion.

Thus, a direct experimental check of existence of «anomalous leptons» of the mass close to $9 \mathrm{MeV}$ still makes sense. 


\section{Photoproduction of «anomalous leptons» in the nucleus Coulomb field}

Our experiment on «anomalous leptons» photoproduction started at the electron synchrotron of the Lebedev Institute in Troitsk. Photon bremmstrahlung beam of $10^{8}$ equivalent photons per sec and the end-point energy in the range 300-500 MeV irradiated $1 \mathrm{~mm}$ copper target. Produced particles $\left(e^{+} e^{-}\right.$and presumably $\left.\ell^{+} \ell^{-}\right)$have been detected by plastic counters at angles near $40^{\circ}$ with respect to the beam. Keeping in mind that time of life of «anomalous leptons» might be only a few ns (including relativistic time delay), the experimental setup was constructed rather compact. Produced positively charged particles were deviated by permanent $1.6 \mathrm{kGs}$ magnets in order to trace particles with momenta near $20 \mathrm{MeV} / \mathrm{c}$ through a few coordinate plastic hodoscopes and organize time-of-flight measurements and velocity separations.

In addition a $20 \times 10 \mathrm{~cm} \mathrm{NaI}(\mathrm{Tl})$ was used in order to measure energy losses of $20 \mathrm{MeV} / \mathrm{c}$ particles in the detector. These energy losses must be rather different for positrons with the kinetic energy $20 \mathrm{MeV}$ and for «anomalous leptons» of the mass $\sim 9 \mathrm{MeV}$ having the kinetic energy closer to $11 \mathrm{MeV}$.

The detection angle of $40^{\circ}$ was chosen to suppress a strong $e^{+} e^{-}$flux of light particles produced at forward angles, whereas the flux of heavier «leptons» is distributed among bigger angles. A Monte Carlo simulation of the Bethe-Heitler pair production (see Fig. 4) illustrates this feature. Our experiment differs from the previous one [5] by using larger registration angle.

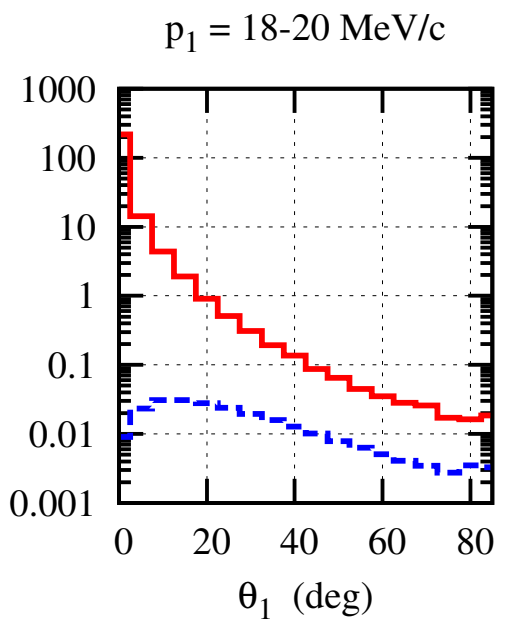

Figure 4. Angular distributions of $e^{+}$(red line) and $\ell^{+}$of the mass $9 \mathrm{MeV}$ (blue line) in the BetherHeitler model. Strong excess of $e^{+}$at very small angles becomes less prominent at angles of order $40^{\circ}$

A layout of the experimental setup is shown in Fig. 5. In Fig. 6 a simulated response of the setup to positrons (left) and «leptons» $\ell^{+}$(center) is shown. On the right panel a response of the $\mathrm{NaI}(\mathrm{Tl})$ detector is shown in one of the test runs. 


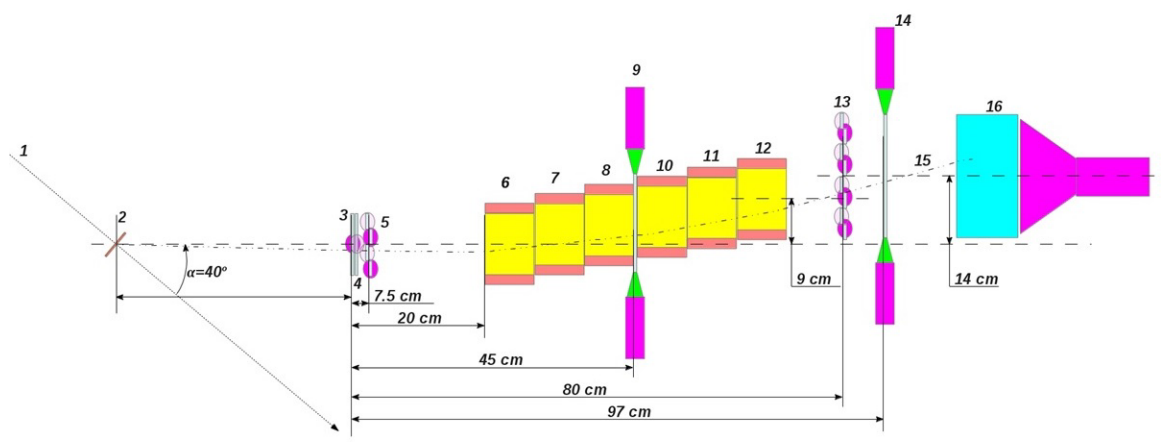

Figure 5. A layout of the setup
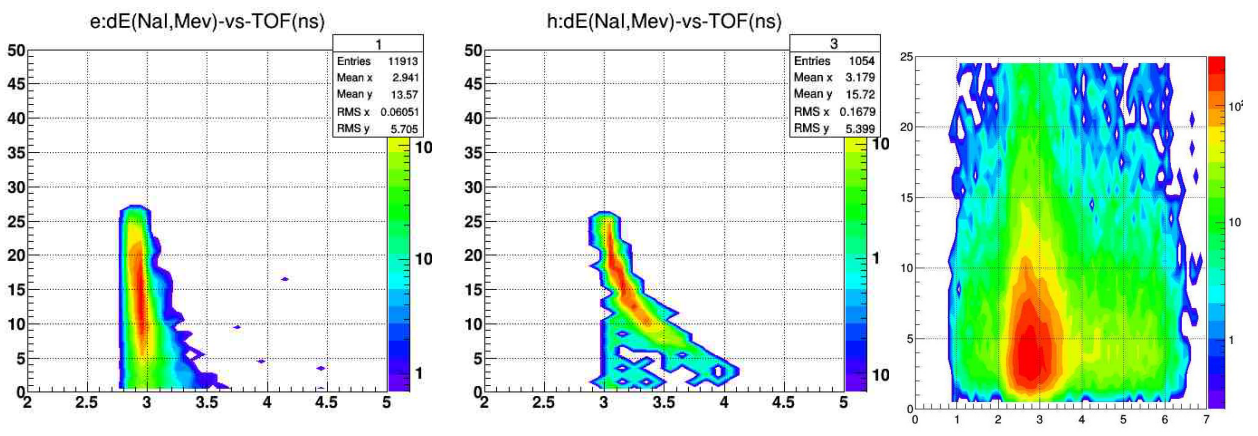

Figure 6. Test run of the setup (right) in comparison with simulations

\section{Conclusions}

The experiment on searching «anomalous leptons» in photoproduction is under way. Chance to find a new physics are minimal. But at least we hope to establish more reliable upper limits for «lepton» production in comparison with older experiments.

\section{References}

[1] V.A. Nikitin, «A search for anomalous lepton» http://museum.jinr.ru/news/153.htm

[2] M. Tanabashi et al. (Particle Data Group), Phys. Rev. D 98, 030001 (2018)

[3] M. Gourdin, E. de Rafael, Nucl. Phys. B 10, 667 (1969)

[4] L.G. Dedenko, G.V. Domogatsky, I.M. Zheleznykh, V.A. Petrunkin, Nucl. Phys. B 57, $263(1973)$

[5] A.S. Belousov et al., Soviet Phys.-JETP 10, 1143 (1960) 